\title{
Memory Modulates Journey-Dependent Coding in the Rat Hippocampus
}

\author{
Janina Ferbinteanu, Prasad Shirvalkar, and Matthew L. Shapiro \\ Dr. Arthur M. Fishberg Department of Neuroscience, Alfred B. and Gudrun J. Kastor Neurobiology of Aging Laboratories, Mount Sinai School of Medicine, \\ New York, New York 10029-6574
}

Neurons in the rat hippocampus signal current location by firing in restricted areas called place fields. During goal-directed tasks in mazes, place fields can also encode past and future positions through journey-dependent activity, which could guide hippocampusdependent behavior and underlie other temporally extended memories, such as autobiographical recollections. The relevance of journeydependent activity for hippocampal-dependent memory, however, is not well understood. To further investigate the relationship between hippocampal journey-dependent activity and memory, we compared neural firing in rats performing two mnemonically distinct but behaviorally identical tasks in the plus maze: a hippocampus-dependent spatial navigation task and a hippocampus-independent cue response task. While place, prospective, and retrospective coding reflected temporally extended behavioral episodes in both tasks, memory strategy altered coding differently before and after the choice point. Before the choice point, when discriminative selection of memory strategy was critical, a switch between the tasks elicited a change in a field's coding category, so that a field that signaled current location in one task coded pending journeys in the other task. After the choice point, however, when memory strategy became irrelevant, the fields preserved coding categories across tasks, so that the same field consistently signaled either current location or the recent journeys. Additionally, on the start arm, firing rates were affected at comparable levels by task and journey; on the goal arm, firing rates predominantly encoded journey. The data demonstrate a direct link between journey-dependent coding and memory and suggest that episodes are encoded by both population and firing rate coding.

\section{Introduction}

In behaving rodents, hippocampal CA1 pyramidal neurons fire in local patches, or place fields, that reflect the rat's location within an environment (O'Keefe and Dostrovsky, 1971). When animals perform goal-directed tasks in mazes, some place fields are active differentially in the same location (Wood et al., 2000; Smith and Mizumori, 2006), depending on journey origin (retrospective coding) or destination (prospective coding) (Frank et al., 2000; Ferbinteanu and Shapiro, 2003). Together, journeyindependent and journey-dependent place fields may provide a temporally extended spatial representation. This type of activity could help support the temporal organization of episodic memory, autobiographical recollections that can also be used to predict future events (Tulving, 1972, 2001, 2002; Tulving and

\footnotetext{
Received March 10, 2011; revised April 15, 2011; accepted May 3, 2011

Author contributions: J.F. and M.L.S. designed research; J.F. performed research; J.F., P.S., and M.L.S. analyzed data; J.F. and M.L.S. wrote the paper.

This work was been supported by NIH Grants AG05138, MH090125, MH065658, and MH073689. We thank Ann Balk for programming, Maojuan Zhuang for assistance with the laboratory work, Geeta Rao for advice on electrophysiological recording, and Eva Pastalkova for discussion of the data.

The authors declare no competing financial interests.

Correspondence should be addressed to Matthew Shapiro, Dr. Arthur M. Fishberg Department of Neuroscience, Alfred B. and Gudrun J. Kastor Neurobiology of Aging Laboratories, Mount Sinai School of Medicine, 1 Gustave L. Levy Place, New York, NY 10029-6574. E-mail: matthew.shapiro@mssm.edu.

Janina Ferbinteanu's present address: Department of Physiology and Pharmacology, SUNY Downstate Medical Center, 450 Clarkson Avenue, Box 29, Brooklyn, NY 11203.

DOI:10.1523/JNEUROSCI.1241-11.2011

Copyright $\odot 2011$ the authors $\quad 0270-6474 / 11 / 319135-12 \$ 15.00 / 0$
}

Markowitsch, 1998; Rosenbaum et al., 2009; Buckner, 2010; Kwan et al., 2010).

The link between hippocampal journey-dependent activity and hippocampal-dependent memory, however, remains unclear. When rats perform a hippocampus-independent spatial alternation task on a modified plus maze, hippocampal place fields on the central stem discriminate between right- and lefthand trials (Wood et al., 2000). If delays are introduced between trials, rendering the task hippocampus-dependent, discriminative firing shifts from the central stem to the enclosure where the rats are confined between trials (Ainge et al., 2007b; Pastalkova et al., 2008). These findings suggest that, rather than being involved in memory for temporally extended behavioral episodes, journey-dependent coding is generated by specific experimental settings (Bower et al., 2005).

To directly test the link between journey-dependent coding and memory, we recorded CA1 activity in rats performing two behaviorally identical but mnemonically distinct tasks in the plus maze (see Fig. 1A). A spatial task required the hippocampus (Ferbinteanu and Shapiro, 2003); a cue approach task did not (Packard and McGaugh, 1996). Both paradigms required an animal to walk from one of a pair of opposite start arms to the goal arm, which contained food. In the spatial memory paradigm, the rat had to remember where food was located. In the cue response paradigm, the rat had to approach a visible cue that indicated food; the location of both cue and food was changed randomly across trials. In the start arms, therefore, the two memory strategies produced different goal-related expectations (e.g., "the food 
is in the east arm" vs "food is by the cue"), and only one supported successful performance in a given trial. In the goal arms, however, after the animals made their choice, the two strategies converged, guiding the animal to approach the selected goal. If journey-dependent coding and memory are directly linked, then journey-dependent coding should be modulated across tasks, particularly on the start arm. We assessed this hypothesis by comparing the activity of CA1 place fields across tasks within the same journey and across journeys within the same task (see Fig. 1B).

\section{Materials and Methods Subjects}

Male Long-Evans rats (300-350 g, 4-6 months old; Charles River Labs) were housed in individual cages ( $12 \mathrm{~h}$ light cycle) and food deprived to $85 \%$ of starting body weight before and during behavioral training and recording. All procedures with animals met NIH guidelines and were approved by the Mount Sinai Institutional Animal Care and Use Committee. Behavioral data are reported from a total of 19 rats: nine received complete hippocampal lesions and 10 served as controls. Electrophysiological data were recorded from a separate group of five rats.

\section{Apparatus}

The plus maze was made of wood, painted gray, and elevated $81.3 \mathrm{~cm}$ from the floor of a room that contained several visual cues. Each of four arms was $59.7 \mathrm{~cm}$ long and $6.4 \mathrm{~cm}$ wide. A gray wooden block $(29.2 \mathrm{~cm}$ high, $6.2 \mathrm{~cm}$ wide, $22 \mathrm{~cm}$ deep) was used to block the start arm that was not used during a trial. A rectangular waiting platform $(32 \times 42 \mathrm{~cm})$ was placed next to the maze. In the cued version of the task, a visible white flag made of wood was used to mark the position of the food on the maze.

\section{Behavioral training and testing}

Rats were trained to walk from either the north or the south start arms to the end of the west or east goal arms to obtain food. The animals were first trained in the cue version of the task, followed by the spatial task when they reached $80 \%$ correct criterion. In the cue version, the start and the goal arm were selected based on a pseudorandom sequence of 60 trials with $\leq 3$ consecutive repetitions of the same type of journey (NE, NW, SE, or SW). This procedure rendered spatial location irrelevant and required a stimulus-response type of behavioral strategy. In the spatial version, the animal had to find food by remembering the spatial location he had recently visited, as previously described (Ferbinteanu and Shapiro, 2003). The location of the food was kept constant until the rat entered the correct goal arm in nine of 10 consecutive trials, when the other goal arm was baited and a new block of trials began. Alternating blocks continued throughout each daily session and included as many as 60 trials. Entry with all four paws into the unrewarded arm defined an error, which the rat was allowed to correct. In both tasks, the animals were placed for $5-10 \mathrm{~s}$ on the side platform between trials. When animals reached criterion of $80 \%$ correct performance in both tasks, they were implanted with 14 tetrodes for neurophysiological recording. During recording, the order in which the animals performed the two tasks was counterbalanced. To verify the neurobiological requirements of the task, two additional groups of animals were trained to a criterion of $80 \%$ correct in either the cue or the spatial navigation task. After reaching criterion, half the animals in each group were given neurotoxic lesions of the hippocampus. Performance on the previously acquired tasks was assessed for $5 \mathrm{~d}$ after recovery from surgery. At the end of behavioral procedures, the animals were overdosed with sodium pentobarbital and the brain tissue processed for histology.

\section{Lesions}

Rats were anesthetized with a small dose of sodium pentobarbital (18 $\mathrm{mg} / \mathrm{kg}$ body weight) administered intraperitoneally, followed by isoflurane. Atropine (5 mg/kg body weight) was also administered to avoid fluid accumulation in the respiratory tract. Neurotoxic lesions were made by injecting a solution of $5 \mathrm{mg} / \mathrm{ml} \mathrm{NMDA}$ in phosphate buffer, $\mathrm{pH}$ 7.4 , at 10 different locations in each hippocampus through a 30 -gauge

\section{Table 1. Lesion coordinates of complete hippocampal lesions}
1. AP, $-3.1 ; \mathrm{L}, \pm 1.0 ; \mathrm{V},-3.6 ; 0.25 \mu \mathrm{l}$
2. AP, $-3.1 ; L, \pm 2.0 ; V,-3.6 ; 0.25 \mu \mathrm{l}$
3. AP, $-4.1 ; \mathrm{L}, \pm 2.0 ; \mathrm{V},-4.0 ; 0.25 \mu \mathrm{l}$
4. AP, $-4.1 ; L, \pm 3.5 ; V,-4.0 ; 0.25 \mu \mathrm{l}$
5. AP, $-5.0 ; \mathrm{L}, \pm 3.0 ; \mathrm{V},-4.1 ; 0.25 \mu \mathrm{l}$
6. AP, $-5.0 ; \mathrm{L}, \pm 5.2 ; \mathrm{V},-5.0 ; 0.25 \mu \mathrm{l}$
7. AP, $-5.0 ; \mathrm{L}, \pm 5.2 ; \mathrm{V},-7.3 ; 0.25 \mu \mathrm{l}$
8. AP, $-5.8 ; \mathrm{L}, \pm 4.4 ; \mathrm{V},-4.4 ; 0.25 \mu \mathrm{l}$
9. AP, $-5.8 ; \mathrm{L}, \pm 5.1 ; \mathrm{V},-6.2 ; 0.40 \mu \mathrm{l}$
10. AP, $-5.8 ; \mathrm{L}, \pm 5.1 ; \mathrm{V},-7.5 ; 0.40 \mu \mathrm{l}$

$\mathrm{AP}$, Anteroposterior from bregma; $\mathrm{L}$, lateral from bregma; $\mathrm{V}$, ventral from bregma. All coordinates are in $\mathrm{mm}$.

cannula attached to a minipump (Harvard). The coordinates of each injection and the volumes injected are presented in Table 1. To prevent seizure development, an intraperitoneal injection of valium $(10 \mathrm{mg} / \mathrm{kg}$ body weight) was administered previous to neurotoxin infusion and animals were monitored until completely awake and active in their home cages. Sham animals were anesthetized, incised, and sutured. Testing resumed $\sim 1$ week after the surgery. Percentage performance error was calculated for each rat during each day of testing. The performance of the groups was compared across days using a day $\times$ lesion group ANOVA (SAS Institute).

\section{Implants}

For electrophysiological recording, 12 tetrodes made from four twisted wires (Ni-Cr wire, Rediohm-800, $12.7 \mu \mathrm{m}$; Kanthal) and two reference wires were loaded into a 14-drive assembly (Neuro-hyperdrive; Kopf Instruments) that allowed independent vertical movement of each drive. The electrode assembly was mounted on the skull with dental cement and bone screws that connected ground wires. The tip of the assembly was lowered to the cortical surface (AP $-3.8 \mathrm{~mm}, \mathrm{ML}-2 \mathrm{~mm}$ from bregma), and at the end of surgery the tetrodes were driven $1.25 \mathrm{~mm}$ into the brain. Testing resumed 1 week after surgery.

\section{Tissue preparation and processing}

Each rat was overdosed with sodium pentobarbital (100 mg/kg, i.p.) and perfused transcardially with normal saline, then $10 \%$ formalin. Coronal sections $(40 \mu \mathrm{m})$ were cut on a freezing-sliding microtome and stained with formol-thionin to highlight cell layers in blue and fiber tracts in red.

\section{Recording methods}

The hyperdrive assembly was mated to a headstage with 54 unity gain, source following amplifiers, and 10 color LEDs for position tracking. Unit signals were differentially amplified (500-3000×), bandpass filtered $(600-6000 \mathrm{~Hz}$ ), digitized (32 points/waveform, $1 \mathrm{~ms}$ sample, $1 \mu \mathrm{s}$ resolution), and stored with LED positions by computer (Cheetah 64 Data Acquisition System; Neuralynx). Waveforms were displayed on a computer screen and played through two audio speakers while the rat was on the platform. The tetrodes were gradually lowered in the CA1 layer 8-10 d after the recovery from surgery, and their tip was positioned based on the configurations of sharp waves and ripples. Unit activity was recorded in one session as the animals performed the cue and the spatial versions of the task successively. The order of the tasks was varied daily and the animal performed a minimum of 45 trials/task. The LED positions $(640 \times 480$ camera pixels, sampled at $30 \mathrm{~Hz})$ were smoothed using a moving average of 5-10 sequentially collected points that occurred within a maximum of $1 \mathrm{~s}$ and $10-20$ camera pixels of one another. When stable and isolable complex spike units were found (Ranck et al., 1982; O'Keefe, 1979; Fox and Ranck, 1981), a recording session ensued that lasted as long as $90 \mathrm{~min}$. If no stable and isolable complex spike units could be found, the tetrodes were advanced $20-30 \mu \mathrm{m}$ and at least $6 \mathrm{~h}$ elapsed before recording to allow the brain tissue to stabilize. Units with spike amplitudes $>100 \mu \mathrm{V}$ and twice the mean noise were discriminated off-line by identifying clusters defined by waveform parameters (Gray et al., 1995). Up to 15 waveforms were separated per tetrode. Because the tetrodes may have shifted overnight even when they were not advanced at 
the end of the recording session, every distinct and isolated waveform was operationally defined as a different unit. Behavioral measures of speed, distance, and direction of movement were calculated from the tracked position of the color LEDs.

\section{Data analysis}

Behavioral correlates of unit activity were compared across tasks or journeys only when the rat's behavior (speed, head direction, time spent, and number of entries per grid unit) was homogenous and statistically indistinguishable in overlapping segments of corresponding journeys (cf. Ferbinteanu and Shapiro, 2003), with a minimum of five trials for each condition.

\section{Place field definition}

Single-unit activity was discriminated off-line by defining elliptical clusters of 8-32 waveform parameters. To define place fields, we used procedures similar to those previously described (Ferbinteanu and Shapiro, 2003). To define place fields, the maze arena was divided into a $56 \times 56$ array of $25 \mathrm{~cm}^{2} /$ grid units. This procedure divided the maze into a similar number of grid units as in our previous analysis (Ferbinteanu and Shapiro, 2003). To verify the robustness of our results, we also used a $48 \times 48$ array of grid units for part of the analysis, setting which best approximated the physical dimensions of the grid units used in our previous study (Ferbinteanu and Shapiro, 2003). The recording session was divided into two subsessions, cue and spatial, corresponding to the type of task the animal performed. Place fields were first identified separately in these two datasets based on overall activity during either of the tasks. This procedure used similar parameters as our previous analysis (Ferbinteanu and Shapiro, 2003) and ensured that place fields active during one task but silent during the other would be included in the pool of cells analyzed. The neural activity was assessed only when the animal crossed a grid unit at least five times moving faster than $2 \mathrm{~cm} / \mathrm{s}$ and the total time in the grid unit was $\geq 300 \mathrm{~ms}$. For each cell, a place field was defined as an area at least four adjacent grid units with mean firing rate $>1$ spike/s with $\geq 5$ spikes per subfield visit. Noncontiguous patches with firing above threshold were defined as subfields. If a cell had a place field on more than one arm, the analysis excluded activity in the center point and treated the cell as having individual subfields on each relevant arm. Only units with an overall firing rate $<2.5 \mathrm{~Hz}$ and subfields on a maximum of three of the four arms were included in the analysis.

\section{Trial separation and behavioral analysis}

Event flags, generated on-line, categorized maze locations (e.g., start of north arm, maze center, end of west arm), marked the beginning and end of each trial, and signaled the entry into the correct goal arm or the occurrence of an error. The event flags identified and sorted each trial into one of five subfiles containing only one of the four types of journeys (e.g., NE, SW, etc.) or error trials. The error trials included frank behavioral errors (when the animal ran to the end of the goal arm that did not contain food on that trial) and alternative trajectories (when the rat entered the incorrect arm before turning immediately and entering the goal arm).

Spatial behavior was then quantified by calculating number of visits normalized by dwell time, movement speed, and the direction of movement in each of the eight cardinal compass point headings for each subfile containing one journey type. Each measure of spatial behavior was calculated for every grid unit, so that one array described the spatial distribution of one parameter across the length and width of each arm with a resolution that approximated the size of the rats' head stage. The array included the length $(\sim 40 \mathrm{~cm})$ of each arm and separately, the area of the choice point, where body turns occurred, and where behavior changed markedly. The array divided the $6.4 \mathrm{~cm}$ width of each arm into two grid units, and included regions where the rat's head could extend past the edge of the arm. Paired $t$ tests assessed whether spatial behavior was equivalent in each pair of corresponding journeys. For example, the north arm visits, head direction, and speed arrays during NE trials were compared with the corresponding arrays for NW trials. A significant difference $(p<0.05)$ in any of the behavioral measures excluded the associated place fields from further consideration. In a second, later stage of analysis, we analyzed patterns of neural activity within and across tasks simultaneously. In this case, we used one-way ANOVA (one for each behavioral parameter) to assess spatial behavior across tasks as well as journeys. For example, the behavior in the north arm was assessed across the NE and NW journeys in the cue task and the NE and NW journeys in the spatial task. A significant difference in any of the behavioral parameters excluded the associated neural activity from further consideration.

\section{Neural activity analysis}

Stage I: across journeys. After place fields were defined in the overall recording file containing data from one task, the data were separated into four subfiles comprised of trials where the animal made the correct behavioral choices, and one subfile that contained the error trials. As in our previous study (Ferbinteanu and Shapiro, 2003), we distinguished between journeys and trajectories. We defined journey as traveling from a start to an end point (from north to east, from north to west, from south to east, and from south to west), which could be accomplished via different paths, or trajectories. Journey-dependent activity was defined as place field firing that showed discriminative activity during different journeys through the same arm (e.g., different firing in the north arm during NE vs NW journeys) regardless of the specific path followed. Note that according to this definition, a place field would be categorized as journey-dependent if it showed either rate- or global-remapping (Leutgeb et al., 2005). Neural activity was assessed using two different methods based on defining place fields and on assessing the distribution of firing rate maps using the Pearson's $r$ and Student's $t$ test. Firing in each subfield defined in the overall file was compared across journeys. Subfield response categories were determined by the presence or absence of the field in both journeys. Journey-independent coding was defined as fields that were present in both journeys: prospective coding as journeydependent activity in the start arms and retrospective coding as journeydependent activity in the goal arms (cf. Ferbinteanu and Shapiro, 2003). We defined weak firing as a place field identified in the overall recording session but not in either of the group of trials containing only specific journeys. Fields whose activity was so sparse that no firing rate statistics could be computed within task (filtered number of spikes was $<10$ total for both subfields) were categorized as not-firing. Continuous statistics compared the firing rate within the arm containing a place field identified in the overall recording (cf. Ferbinteanu and Shapiro, 2003). Firing rates were calculated by dividing the total number of spikes by the total amount of time spent in each grid unit. Each maze arm was represented by a $3 \times 10$ (start arm) or $3 \times 12$ (goal arm) array of grid units. The start arm array was shorter than the goal arm array to account for the rat's starting orientation facing the choice point in the start arm. The spatial distribution of firing rates along an arm was compared across journeys using Pearson's $r$. The mean firing rates within the arm was compared across journeys using Student's $t$ test. A field was considered journeydependent if either the $t$ test indicated a significant difference in the mean firing rates or if the Pearson's $r$ was statistically not different from 0 (Howell, 1997). The field was otherwise assigned to either journeyindependent (when the $t$ test and correlations could be calculated) or to the not-firing category. Note that using $t$ test/Pearson's $r$ statistics entailed that there was no weak firing category.

Stage II: across tasks. To assess changes in patterns of neural activity across tasks, we included only activity recorded during sessions with a sufficient number of trials in both tasks and with no differences in the behavioral parameters across the four corresponding journeys. With the subfield count method, a subfield was considered to encode the memory strategy if the field was present in one journey but not the other across tasks. For the firing rate method, Pearson's correlation coefficients and Student's $t$ tests were calculated as described above; similarly, a field was considered to encode memory strategy if ether the $t$ test indicated a significant difference in the mean firing rates or Pearson's $r$ was statistically not different from 0 . However, in this case, fields inactive in both journeys were considered to have had highly correlated firing rate maps and similar firing rates.

\section{Error trials analysis}

For a field to be considered in the error analysis, the rat had to make at least two incorrect trajectories through the region that included the 
journey-dependent place field. For each journey-dependent place field, the journey type that had a field was designated as the field's preferred journey and the corresponding journey that had no place field was designated nonpreferred. A field was considered assessable if it included at least four error trials (mean $=7$ ); all but three cells had more than two trials in both the preferred and nonpreferred journeys. Assessable fields were categorized as losing or maintaining selectivity during error trials. If the unit was significantly active in at least two trials (and $\geq 50 \%$ of the incorrect trajectories) during nonpreferred journeys, then the field was categorized as losing selectivity. Three fields were also classified as losing selectivity because the units stopped firing altogether during preferred, but erroneous, journeys. A field was categorized as maintaining selectivity during errors if it was active in at least one of two or more preferred journeys and in no more than one of four or more nonpreferred journeys. Most (73 of 94) of the assessable fields had all-or-none activity patterns during correct and incorrect journeys. The place fields that distinguished journeys by different mean firing rates (21 of 94 fields), were assessed as above by comparing relative firing rates during error and correct trials.

\section{Cell-wise ANOVA analysis}

To concomitantly evaluate the effects of spatial location, task, and journey-type on firing rate of individual cells, we computed a three-way ANOVA using trial-wise firing rate as the dependent variable and task (cue vs spatial), journey (start arms: NE vs NW and SE vs SW; goal arms: NE vs SE and NW vs SW), and spatial location $(2 \mathrm{~cm}$ bin units along the linearized track) as the independent variables. The ANOVA models were constructed with main effects and a full combination of all interactions. For each arm, we computed the number and percentage of cells with a significant main effect or interaction at the level of the entire population of cells. Combinations of main effects were considered only if the field did not have an interaction of the same effects. For example, a field would be considered to show a task-and-journey effect only if it did not have a task $\times$ journey significant interaction. For each main effect, interaction and conjunction, we also counted the number of neural ensembles on the start and goal arms that included at least one cell showing the corresponding effect.

\section{Firing rate function analysis}

To evaluate the changes in overall activity across journeys and tasks as animals walk on the maze (which encompasses the activity of both journey-dependent and-independent fields), we calculated a spatial rate function for the collection of fields simultaneously active in a given arm, referred to here as assemblies. All analysis and programming was done in MATLAB 7.7 (MathWorks). The function was formed by computing a bin-wise firing rate for each field of an assembly in each condition (see Fig. $5 A$ ). Firing rates were obtained by dividing the number of speedfiltered $(2 \mathrm{~cm} / \mathrm{s})$ spikes recorded in all trials of a journey by the occupancy time of the rat in that bin (over all trials). The path of the rat for each journey was linearized by projecting the actual trajectory followed by the animal on that trial onto a user-defined idealized path. The projection was determined using nearest neighbor Delaunay triangulation (dsearchn function in MATLAB). Spatial bins had a resolution of $2 \mathrm{~cm}$. The first and last spatial bins on the track were omitted to remove edge artifacts (bin 1, beginning of start arm; bin 40, end of goal arm). For pseudocolor plots of ensemble firing functions, the order of cells for display was determined by sorting the firing rate functions by the spatial location of the maximum. Cells were organized from bin 1 to bin 38 in an ascending manner.

\section{Rate difference analysis}

We computed difference plots between each ensemble spatial rate functions for similar journeys across tasks (cue vs spatial) and for pairs of journeys within task with the same start or goal points (see Fig. 5B). For each assembly, the magnitude of firing rate difference in one location was computed by adding the absolute values of differences belonging to that bin (see Fig. 5C). Firing rate differences for each neural assembly in each spatial bin were averaged to compute the mean effects of task and journey on firing rates along the start and goal arms (see Fig. 5D). Because behavior in the choice point was similar across tasks but different across jour- neys, the firing rate functions in the center point could be used to assess only the influence of memory strategy. The actual results were compared statistically to a shuffled firing rate distribution obtained by mixing condition identity.

\section{Group statistics and shuffling}

Group statistics for the spatial rate function differences were computed by averaging over each assembly summed spatial difference. Ninety-five percent confidence intervals for the difference plots were computed for each spatial bin by bootstrapping the ensemble averages within each spatial bin 1000 times. We compared the spatial difference functions between tasks or journeys with those of trial-shuffled datasets by task or journey identity, respectively. For across-task shuffling, the task identity of individual trials on a single journey were randomly assigned to either task (cue or spatial). New surrogate cue or spatial session-averaged data were then created by independently averaging over shuffled trial data. For across-journey shuffling, the journey identity of individual trials sharing a common arm within a task (i.e., NE, NW) were randomly assigned. New surrogate Journey1 or Journey2 session-averaged data were then created by independently averaging over shuffled trial data. The task-shuffled or journey-shuffled mean and confidence intervals were computed as above.

\section{Population vector analysis}

For each neural assembly, we computed the correlation between population vectors across tasks when the animals followed similar journeys and across journeys with the same start or goal point within the same task. Each population vector consisted of the totality of bin-wise firing rates of all the fields belonging to a neural assembly (the collection of fields simultaneously active in a given arm). The degree of correlation between pairs of population vectors was assessed by computing the Pearson's $r$ between the corresponding elements of the two firing rate functions. The Pearson's $r$ was then converted to Fisher's $z$ for statistical comparisons across groups.

\section{Results}

Hippocampal activity was necessary for spatial memory, but not cue approach performance

To demonstrate differences in the neural substrates required for each tasks, we first tested the effects of hippocampal neurotoxic lesions on task performance. Sham animals continued to perform well after the surgery. Animals with hippocampal lesions were motivated, entered arms readily, and performed the cue task normally, but were impaired severely and selectively in the spatial task (ANOVA for the spatial task: lesion: $F_{(1,6)}=51.84, p<0.001$; day: $F_{(6,36)}=19.37, p<0.001$; lesion $\times$ day: $F_{(6,36)}=14.77, p<$ 0.001; ANOVA for the cue task: lesion $F_{(1,9)}=0.04, p=0.84$; day: $F_{(6,54)}=7.82, p<0.001$; lesion $\times$ day: $\left.F_{(6,54)}=1.59, p=0.1668\right)$. This experiment confirmed that hippocampal neurons are necessary for performing the spatial memory, but not cue approach version of the plus maze task (Fig. 2A). The analysis included only animals with minimal cortical damage, complete damage to the dorsal hippocampus, and substantial damage to the ventral hippocampus (Fig. $2 B$ ).

\section{Proportions of prospective and retrospective fields were similar in both tasks, but memory modulated hippocampal activity}

Many cells fired on more than one arm; some were active only in one task and others were active in both. Because multiple fields belonging to the same unit showed independent patterns of activity within and across tasks (Fig. 1D), each subfield was analyzed separately. We first identified place fields separately in each task, and found equivalent numbers of place fields in both. In the spatial task, we recorded 1022 fields (447 start arm and 575 goal arm); of these, 648 were subfields of the same unit. In the cue task, 

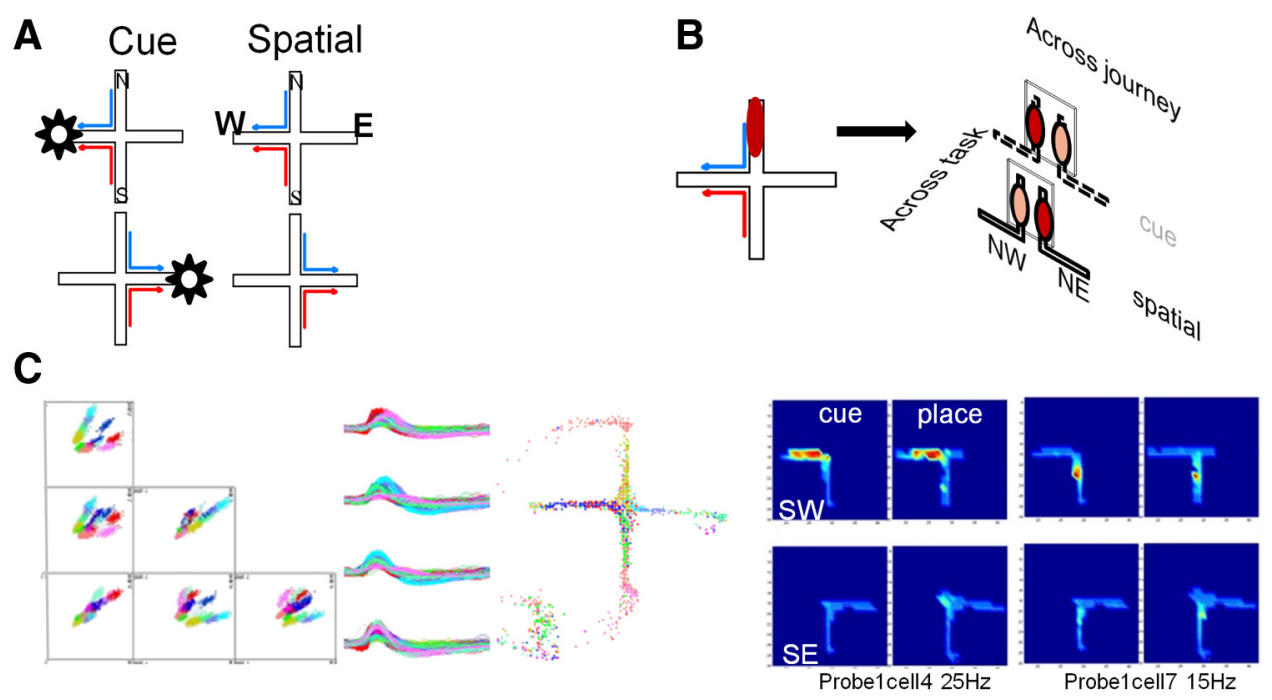

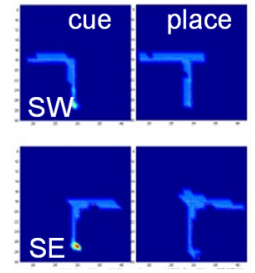

Probe1cell14 $18 \mathrm{~Hz}$
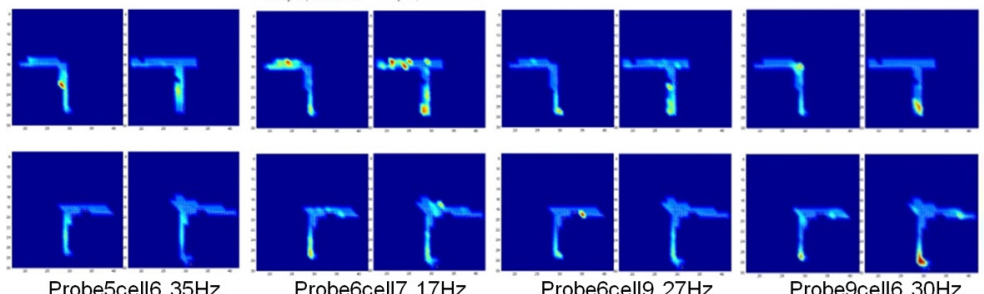

D
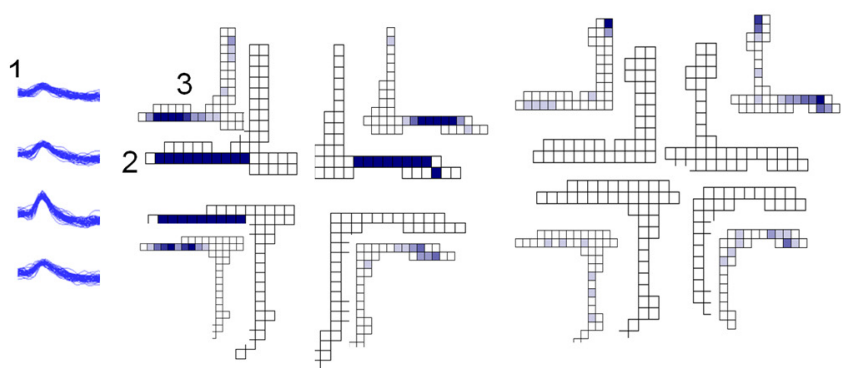

Figure 1. Experimental design and firing patterns across tasks and journeys. A, Tasks. Rats were trained to walk from either the north or south start arms to either the east or west goal arms in a plus maze. In the cue approach task, food location was signaled by a visible cue at the end of a goal arm, the location of which varied pseudorandomly across trials (left). In the place approach task, rats were trained to enter either the east or west goal depending on recent memory (right). $\boldsymbol{B}$, Data parsing. Neural activity across tasks and journeys was quantified by identifying place fields in either the cue or the spatial segment of a recording session. The spatial distribution of firing rates for each field was quantified separately for each condition, giving four rate maps, each map corresponding to one journey in one task [e.g., a place field in the north arm (left) was characterized during NE and NW journeys in the place and cue tasks (right)]. Place field analyses only included the areas where the behavior of the animal was homogenous across conditions (rectangles). C, Examples of place fields recorded in a session that included both cue and spatial tasks. Top, left, The six amplitudeto-amplitude projections (left), waveforms (middle), and firing location (right) of all 15 units recorded on one of the 10 active tetrodes during this session. Place fields (on a blue background) show examples of seven cells with activity in the south start arm parsed into four firing rate maps, each corresponding to a unique combination of task (cue, place) and journey (SE, SW). The maps show a small population of neurons distinguished both task and journey. The normalized firing rate is shown by color, with the maximum firing rate in the south start arm noted below each map. $D$, Measurement methods. Differences in activity were assessed by counting subfields and comparing firing rate maps. The waveform of the unit in this example $(1$, waveform max $=288 \mu \mathrm{V})$ is on the left. The subfield maps (2) and firing rate maps (3) are shown for each journey in the cue (middle) and spatial (right) tasks. Shading indicates levels of activity (lighter color, lower firing rate). In the cue task, the field had strong journey-independent activity in the west arm ( $p$ value of Student's $t$ test $=0.21$, Pearson's $r=0.33$ ) and journey-dependent activity in the east arm ( $p$ value of Student's $t=0.03$, Pearson's $r=0.57$ ). In the spatial task, the same unit fired weakly (west: ip value of Student's $t=0.74$, Pearson's $r=0.30$; east: $p$ value of Student's $t=0.05$, Pearson's $r=0.05$ ).

we recorded 1084 fields, (539 start arm and 545 goal arm); of these, 766 were subfields of the same unit. Thus, the overall level of hippocampal activity was not affected by task demands. To directly compare the firing patterns between the tasks, we selected the fields with sufficient sampling in both tasks. Of the 646 fields that met these criteria, 259 were highly active during both tasks, 204 were active only in the cue task, and 183 were active only in the place task. Hippocampal place field activity thereby reflected the different task demands even as rats performed identical behaviors in the same environment. The activity of these fields was analyzed individually and in 65 neural assemblies formed by the neural population recorded simultaneously in a given area of the maze (2-29 units; median, 9) [32 assemblies on the start arm (327 fields) and 33 assemblies on the goal arm (319 fields)].

We hypothesized that journey-dependent coding revealed a neuronal signature that reflected situations in which the hippocampus guided ongoing behavior. Based on previous work (Frank et al., 2000; Bower et al., 2005; Kim and Frank, 2009), we predicted lower or no journey-dependent activity, particularly prospective coding, in the hippocampal-independent task. In contrast to our prediction, the proportion of journey-dependent fields was similar across tasks by every measure (start arm: sub- 


\section{A Spatial navigation}

\section{Cue response}

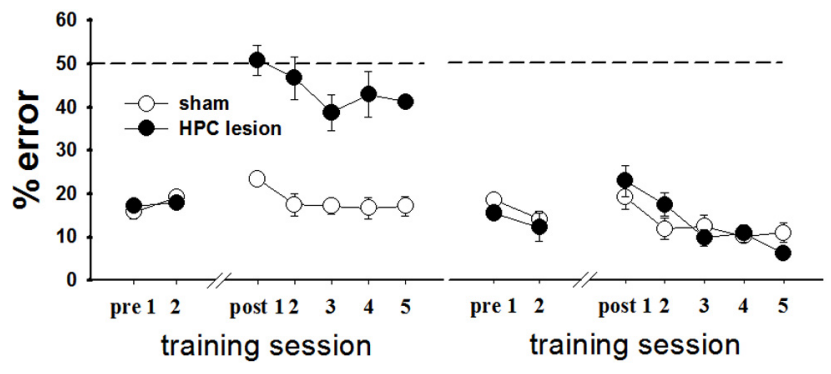

B
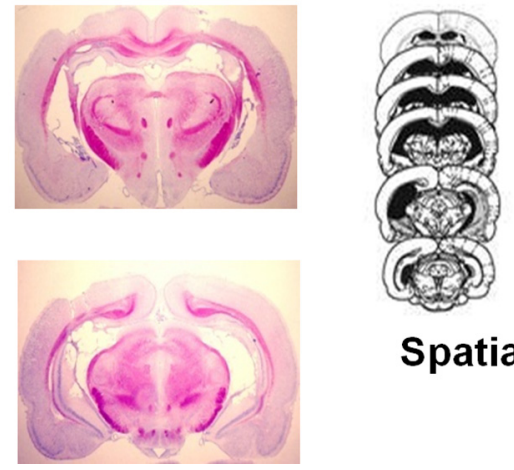

Spatial

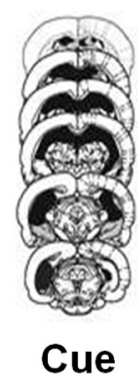

Figure 2. Hippocampal activity was necessary for spatial memory, but not cue approach performance. $A$, After reaching a criterion of $80 \%$ correct on two consecutive days, the rats were given complete neurotoxic hippocampal lesions or sham surgery $(n=10)$. Rats with lesions performed poorly in the spatial navigation task ( $n=5$, left), but normally in the cue approach task ( $n=4$, right). Dashed lines at the $50 \%$ mark indicate random level performance. $\boldsymbol{B}$, Lesions encompassed dorsal hippocampus and large portions of the ventral hippocampus. Left, Representative hippocampal lesion; right, minimum (black) and maximum (gray) extents of the lesions in the two experimental groups.

field count: $\chi_{(1)}^{2}=1.3, p=0.25$; firing rate: $\chi_{(1)}^{2}=1.37, p=$ 0.24 ; goal arm: subfield count: $\chi^{2}{ }_{(1)}=2.45, p=0.11$; firing rate: $\chi_{(1)}^{2}=2.8, p=0.09$ ) (Fig. $3 A$ ), suggesting that at least in certain conditions, the hippocampus is always on-line (Morris and Frey, 1997) and codes memory-based discriminations even when the structure is not required for ongoing memory performance. As described before, retrospective coding was more common than prospective coding in both tasks (subfield counts: cue: $\chi^{2}{ }_{(1)}=$ 18.07, $p<0.0001$; spatial: $\chi_{(1)}^{2}=14.52, p=0.0001$; firing rate: cue: $\chi_{(1)}^{2}=100.17, p<0.0001$; spatial: $\chi_{(1)}^{2}=56.91, p<$ $0.0001)$. The same distribution emerged for the more inclusive datasets of 1084 and 1022 place fields recorded in the cue and spatial task, respectively (Tables 2, 3), and it was the same as in the previous study, when the animals were trained and performed a spatial task only (Ferbinteanu and Shapiro, 2003).

Prior work found that journey coding diminished when rats made spatial memory errors (Ferbinteanu and Shapiro, 2003), suggesting that the hippocampal signal helped to discriminate among recent episodes. We expected to replicate that result in the spatial task and predicted that changes to journey coding would be unrelated to errors in the cue task. In fact, journey-dependent coding did diminish when rats made errors, but to the same degree in both tasks. Of the 62 fields that could be assessed in the cue task and 108 fields that could be assessed in the spatial task during error trials, most lost differential firing (as measured by one or both methods of activity assessment) when the animal entered the wrong arm (cue: $\chi_{(1)}^{2}=16.51, p<0.0001$; spatial: $\chi_{(1)}^{2}=29.03, p<0.0001$ ) (Fig. 3B). Though prospective coding appeared to be more affected than retrospective, the differences

were not significant (prospective vs retrospective: cue: $\chi^{2}{ }_{(2)}=$ 5.06, $p=0.07$; spatial: $\chi_{(2)}^{2}=4.36, p=0.11$ ). The results suggest that although the hippocampus is not required to perform the cue approach task, journey-dependent activity may still be linked to performance.

Despite the similar proportions of journey-dependent activity found in the two tasks, memory strategy modulated the activity of individual fields (Fig. 3C), a phenomenon observed across maze areas and place field categories. Continuous measures were more sensitive to task change than categorical statistics, indicating that hippocampal neurons responded to a switch in memory strategy with altered firing rates akin to rate remapping rather than in the all-or-none fashion described as global remapping (Leutgeb et al., 2005). This configuration of results suggests that, although change in activity pattern is defined by change in firing rates, firing rates and journey-dependent activity patterns may not be identical neural codes (Fig. 3D). Consequently, in the subsequent analysis, we investigated the effect of change in memory strategy on both journey-dependent activity pattern and firing rates more generally.

\section{Active selection of memory strategy modulated prospective coding}

The current experiment investigated the link between memory and journey-dependent coding by recording activity in two behaviorally identical, but mnemonically distinct behavioral tasks. The task structure ensured that in the start arm, but not the goal arm, the animals had to actively select between two memory strategies that could not be interchanged to support successful performance. Thus, if memory modulates journey-dependent coding, it should predominantly influence prospective coding. Although our initial prediction was not confirmed, we found that in the start arms, $\sim 50 \%$ of the place fields changed coding category between tasks, so that a field showing prospective coding in one task was as likely to become journey-independent as it was to maintain prospective coding in the other task, and vice versa. In the goal arm, however, $\sim 84 \%$ of the place fields maintained retrospective coding in both tasks, a significantly greater proportion than in the start arm (cue: $\chi_{(1)}^{2}=76.90, p<0.0001$; spatial: $\chi_{(1)}^{2}=135.74, p<0.0001$ ) (Fig. $4 A$ ). Table 4 enumerates the coding categories changes across tasks in all units that were recorded in both tasks. The entries along the diagonals (bold) indicate the number of units that maintained the same coding category across tasks (e.g., were journey-independent in both the place and cue tasks). The remaining entries show differential coding in the two tasks. For this analysis, the effect of task changes on place field coding category was measured by the continuous statistics described above. Because the overall proportions of journey-dependent fields did not change across the tasks, the results suggest that journey destination is coded by different neuronal populations across tasks, whereas journey origin is not. This result further implies that the subpopulation of neurons showing prospective coding include more information about memory strategy than the subpopulation showing retrospective coding.

Firing rates of journey-dependent fields encoded predominantly journey origin when memory strategy was irrelevant

To directly compare the relative influence of memory strategy and journey type on firing rates of journey-dependent CA1 fields, we investigated the effect of location, journey, and task in a re- 


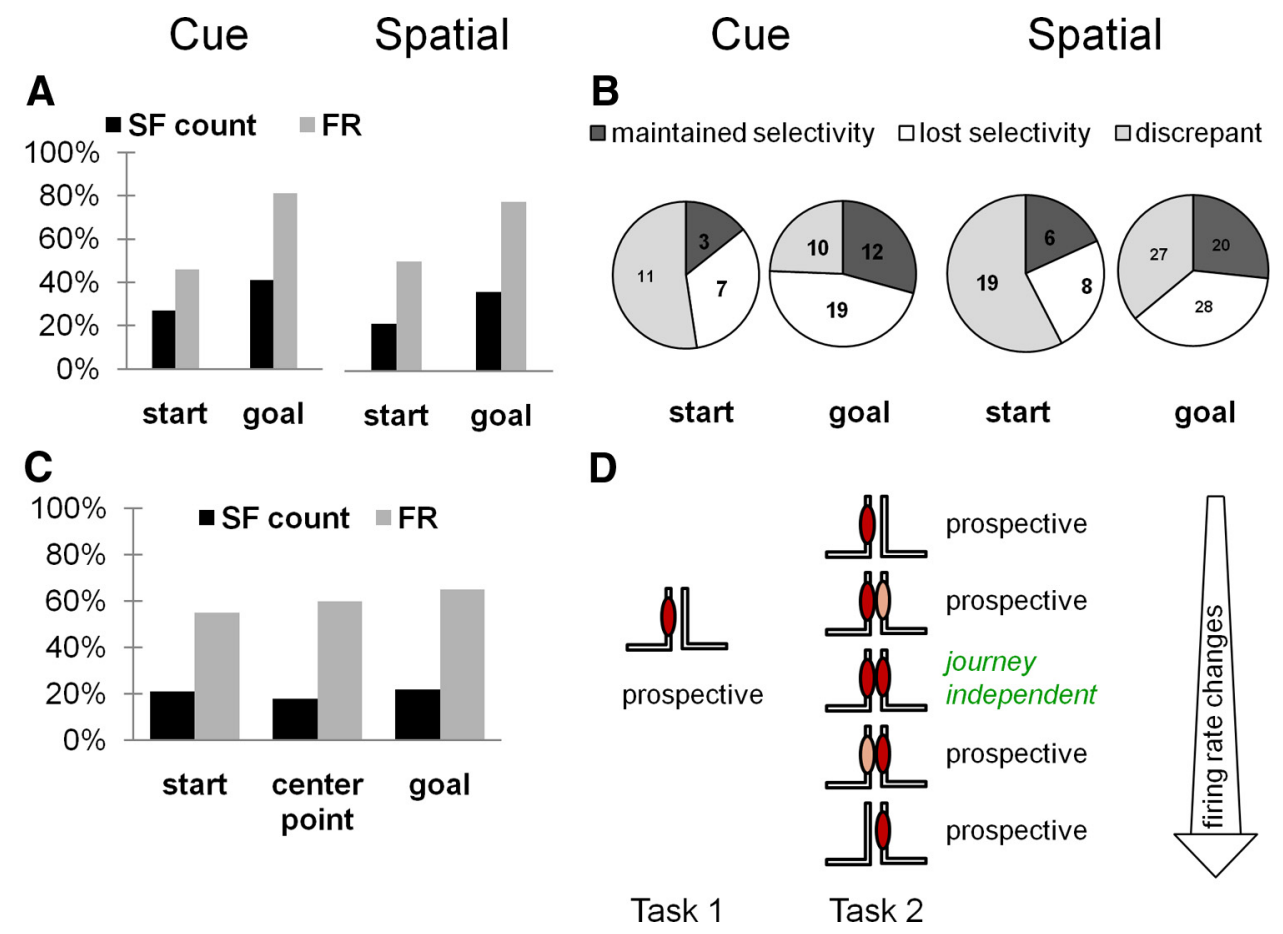

Figure 3. Proportions of journey-dependent place fields were similar in the two tasks, even as memory strategy modulated journey-dependent activity. A, Journey-dependent signal. Proportions of journey-dependent fields were similar in the cue and place approach tasks, and retrospective coding was more common than prospective coding in both. Subfield count (SF) categorized the presence or absence of a field in a given journey; firing rate (FR) within the arm quantified the spatial distribution and mean firing rate using Pearson's $r$ and Student's $t$ tests. $\boldsymbol{B}$, Journey coding during errors trials. Most journey-dependent fields lost discriminative activity during error trials. Fields that maintained selectivity according to both subfield count and firing rate analysis methods are shown in dark gray, fields that lost selectivity according to one of the methods are shown in light gray, and fields that lost selectivity according to both methods are shown in white. Relative to the total number of place fields recorded, few could be assessed during error trials, particularly in the cue response task, as the numbers inside of the pie charts indicate. C, Memory strategy effect. Memory strategy modulated the activity of place fields in all maze areas mainly by altering firing rates along the arm (firing rate, gray) rather than causing an all-or-none change (subfield count, black). $\boldsymbol{D}$, Journey-dependent coding and firing rate. Because journey-dependent coding is defined categorically by a statistical threshold, it encompasses a wide range of potential firing rate changes. For example, a field showing prospective activity by firing exclusively in one journey during one task (north to west, left) can continue to show prospective activity in the other task in widely different activity patterns (right). Whether the cell continues to fire exclusively in the same (north to west) journey, the opposite (south to west) journey, or in both journeys but at different rates, prospective coding is maintained. Thus, proportions of journey-dependent fields can remain constant across tasks ( $\boldsymbol{A}$ ) even as their activity patterns discriminate task demands ( $(\boldsymbol{)}$.

\section{Table 2. Activity patterns of fields recorded in the cue task}

\begin{tabular}{lr}
\hline Subfield count & \\
Journey-independent & 220 \\
Prospective & 145 \\
Retrospective & 264 \\
Weak & 393 \\
Did not fire & 62 \\
Grand total & 1084 \\
Firing rate & \\
Journey-independent & 324 \\
Prospective & 243 \\
Retrospective & 455 \\
Did not fire & 62 \\
Grand total & 1084 \\
\hline
\end{tabular}

peated measures three-way ANOVA. Each place field was analyzed separately in a model that used location (bin), journey, and task to predict trial-wise firing rate (Fig. $4 B$, Table 5). As expected of place fields, location (bin) significantly affected firing rates. Independent of this effect, journey and task also affected firing rates, and the effects differed in the start and goal arms. In the start arm, task and journey modulated a similar number of place fields (task vs journey: cue task: $\chi_{(1)}^{2}=0.00, p=1.00$; spatial task: $\left.\chi_{(1)}^{2}=1.03, p=0.31\right)$. In the goal arm, however, journey origin affected a significantly higher proportion of place fields than task (task vs journey: cue: $\chi_{(1)}^{2}=9.73, p=0.001$; spatial: $\left.\chi_{(1)}^{2}=14.01, p=0.002\right)$. The same pattern of results was ob-
Table 3. Activity patterns of fields recorded in the spatial task

\begin{tabular}{lr}
\hline Subfield count & \\
Journey-independent & 203 \\
Prospective & 116 \\
Retrospective & 253 \\
Weak & 402 \\
Did not fire & 48 \\
Grand total & 1022 \\
Firing rate & \\
Journey-independent & 287 \\
Prospective & 226 \\
Retrospective & 461 \\
Did not fire & 48 \\
Grand total & 1022 \\
\hline
\end{tabular}

tained when interactions or combinations with spatial location were included (location $\times$ task and location and task combined; start arm: cue: $\chi_{(1)}^{2}=2.8, p=0.09$; spatial: $\chi_{(1)}^{2}=0.00, p=1.0$; goal arm: cue: $\chi_{(1)}^{2}=32.05, p<0.0001$; spatial: $\chi_{(1)}^{2}=34.00$, $p<0.0001)$. Most units that were significantly affected by both task and journey, either as an interaction or as a combination of main effects, were in the goal arm. The effects were well distributed across animals and recording sessions (Table 6). In summary, task coding was more prominent when memory strategy was needed to guide discriminative responses. In contrast, journey coding was more prominent when memory strategy was no longer needed to guide response selection. 
A
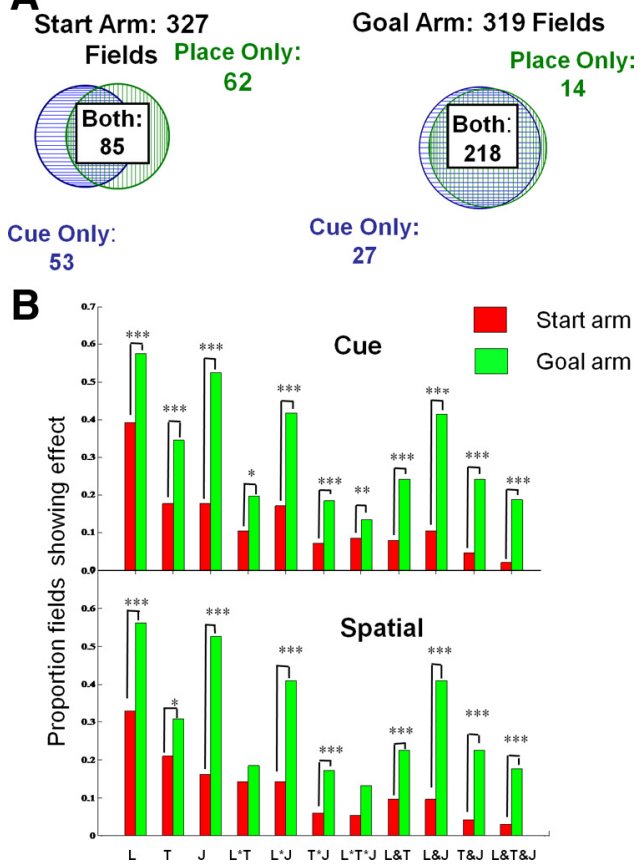

Figure 4. Memory strategy differentially affects fields with prospective and retrospective activity. $A$, Population coding. The total number of place fields in the start and goal arms is listed above each Venn diagram, and the number of journey-dependent fields in each task is indicated by color text. The Venn diagrams show the numbers of place fields that were journeydependent in either the place (green vertical lines), cue (blue horizontal lines), or both tasks (hatching). In the start arm, only 85 fields showed prospective coding in both tasks (left), compared with 218 fields in the goal arm that had stable retrospective coding across tasks (right). The diagrams do not include the place fields that were inactive in either or both tasks. The total number of place fields recorded in start and goal arms was approximately the same. $\boldsymbol{B}$, Firing rate coding. Spatial location (L), task (T), and journey (J) modulated firing rates of prospective (red) and retrospective (green) fields. Firing rates in the start arms were equally affected by memory strategy and journey destination, whereas in the goal arms, rates were most strongly influenced by journey. Activity in each field was analyzed separately across repeated trials. Because the fields with journey-dependent activity were not the same in the two tasks, the data show separately the results on the discriminative fields in the cue (top) and spatial (bottom) tasks. In $x$-axis labels: ${ }^{*}$, interaction effects; $\&$, combinations without interactions. Vertical axes show proportions of journey-dependent fields showing the effects. ${ }^{*} p<0.05$; ${ }^{* *} p<0.01 ; * * * 0.001$.

Table 4. Coding category changes in place fields across cue and place tasks

\begin{tabular}{lrrrrr}
\hline & \multicolumn{2}{l}{ Cue task } & & & \\
\cline { 2 - 6 } Place task & J & DNF & RET & PRO & Total \\
\hline Goal arm & & & & & \\
JI & $\mathbf{1 3}$ & 0 & 27 & - & 40 \\
DNF & 0 & $\mathbf{1 4}$ & 16 & - & 30 \\
RET & 14 & 17 & $\mathbf{2 1 8}$ & - & 249 \\
Subtotal & 27 & 31 & 261 & - & 319 \\
Start arm & & & & & \\
Jl & $\mathbf{6 7}$ & 1 & - & 53 & 121 \\
DNF & 6 & $\mathbf{1 8}$ & - & $\mathbf{8 5}$ & 39 \\
PRO & 62 & 20 & - & 153 & 327 \\
Subtotal & 135 & 39 & - & & 167 \\
\hline
\end{tabular}

$\mathrm{Jl}$, Journey-independent; PRO, prospective; RET, retrospective; DNF, did not fire.

Hippocampal assemblies encoded memory strategy more in the start arm and journey more in the goal arm

To quantify how memory variables altered firing rates as the rats traversed the maze, we compared the mean firing rates across tasks and journeys in the 65 neural assemblies constituted of simultaneously recorded place fields. For each of the four condi-
Table 5. Number of journey-dependent fields encoding spatial location, task and journey, or interactions and combinations of those in the start and the goal arms

\begin{tabular}{lrrrc}
\hline & Start & Goal & $\chi^{2}{ }_{(1)}$ & $p$ \\
\hline Cue task & & & & \\
Location & 60 & 150 & 12.86 & 0.0003 \\
Task & 27 & 90 & 13.48 & 0.0002 \\
Journey & 27 & 137 & 48.95 & $<0.0001$ \\
Location $\times$ Task & 16 & 51 & 5.86 & $0.01^{*}$ \\
Location $\times$ journey & 26 & 109 & 26.92 & $<0.0001$ \\
Task $\times$ journey & 11 & 48 & 9.90 & 0.0016 \\
Location $\times$ task $\times$ journey & 13 & 35 & 2.27 & 0.13 \\
Location and task & 12 & 63 & 17.26 & $<0.0001$ \\
Location and journey & 16 & 108 & 43.95 & $<0.0001$ \\
Task and journey & 7 & 63 & 26.27 & $<0.0001$ \\
Location and task and journey & 3 & 37 & 16.49 & $<0.0001$ \\
Insufficient firing & 3 & 49 & 24.82 & $<0.0001$ \\
Spatial task & & & & \\
Location & 55 & 140 & 21.77 & $<0.0001$ \\
Task & 35 & 77 & 5.04 & $0.02^{*}$ \\
Journey & 27 & 131 & 48.56 & $<0.0001$ \\
Location $\times$ task & 24 & 46 & 1.2 & 0.27 \\
Location $\times$ journey & 24 & 102 & 33.47 & $<0.0001$ \\
Task $\times$ journey & 10 & 43 & 11.44 & 0.0007 \\
Location $\times$ task $\times$ journey & 9 & 33 & 6.81 & 0.009 \\
Location and task & 16 & 56 & 11.63 & 0.0006 \\
Location and journey & 16 & 102 & 48.45 & $<0.0001$ \\
Task and journey & 7 & 56 & 26.04 & $<0.0001$ \\
Location and task and journey & 5 & 44 & 20.72 & $<0.0001$ \\
Insufficient firing & 6 & 13 & 0.6 & 0.43 \\
\hline
\end{tabular}

Start: 153 fields for cue task and 167 fields for spatial task. Goal: 261 fields for cue task and 249 for spatial task.

Table 6. Number of assemblies encoding spatial location, task and journey, or combinations of those in the start and the goal arms

\begin{tabular}{lllll}
\hline & $\begin{array}{l}\text { Start arm } \\
(32 \text { assemblies })\end{array}$ & $\begin{array}{l}\text { Goal arm } \\
(33 \text { assemblies })\end{array}$ & $\chi_{(1)}^{2}$ & $p$ \\
\hline Location & 30 & 32 & 0.38 & 0.53 \\
Task & 27 & 31 & 1.54 & 0.21 \\
Journey & 21 & 31 & 8.14 & 0.004 \\
Location $\times$ task & 22 & 26 & 0.84 & 0.35 \\
Location $\times$ journey & 21 & 30 & 6.14 & 0.01 \\
Task $\times$ journey & 19 & 21 & 0.12 & 0.72 \\
Location $\times$ task $\times$ journey & 13 & 24 & 6.82 & 0.09 \\
Location and task & 12 & 22 & 5.54 & 0.01 \\
Location and journey & 11 & 22 & 7.33 & 0.007 \\
Task and journey & 5 & 19 & 12.27 & 0.0005 \\
Location and task and journey & 6 & 22 & 15.21 & $<0.0001$ \\
\hline
\end{tabular}

tions, the bin-wise firing rates of each field in an assembly were aligned spatially to define a firing rate function along the length of each journey (see Materials and Methods, above) (Fig. 5A). To compare ensemble responses to different tasks and journeys, the values in corresponding spatial bins were subtracted to produce four firing rate difference functions (two across journeys, two across tasks) (Fig. 5B,C). In the start arms, memory strategy influenced assembly firing rates more, but at comparable levels as journey destination (location $\times$ condition, start arm: $F_{(16,1008)}=$ $1.71, p=0.04)$ (Fig. 5D, red vs black lines). In the goal arms, in contrast, the origin of the journey had greater influence on ensemble codes than memory strategy (location $\times$ condition goal arm: $\left.F_{(16,1040)}=9.4, p<0.0001\right)$. The relative influence of journeys was particularly strong as the rat exited the choice point (Fig. $5 D$, green vs black lines). Overall, assembly firing rates were more distinct in the goal than in the start arms, whether compared across tasks or journeys (start vs goal arm: tasks: $F_{(1,2612)}=16.95$, 
A

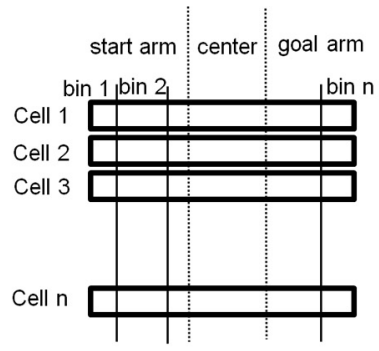

B
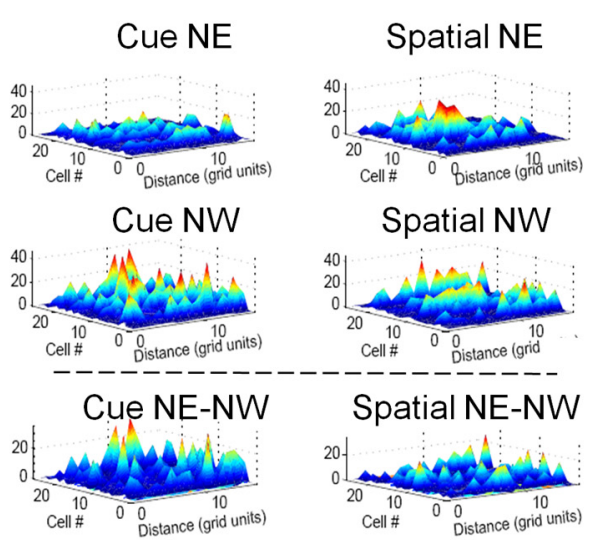

journey

\section{C}
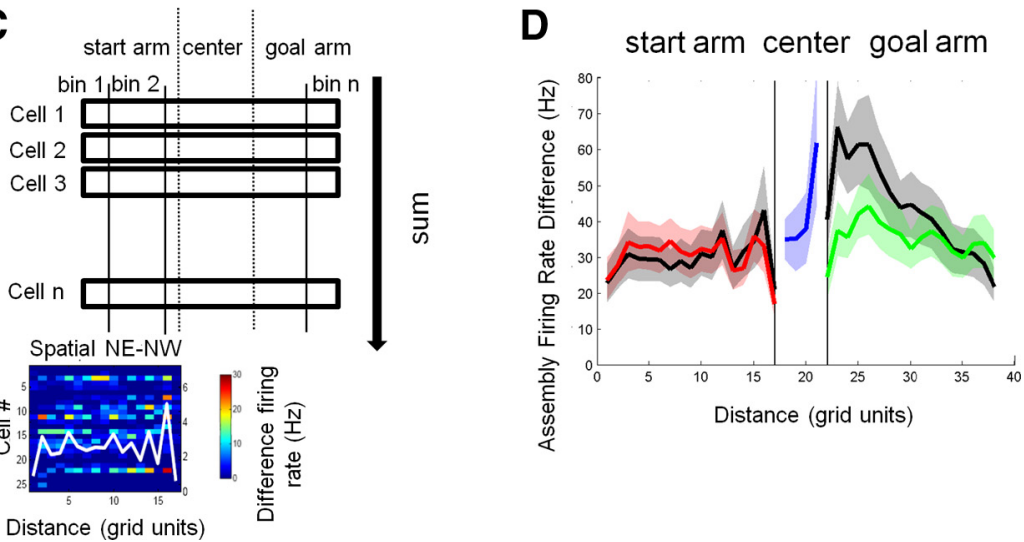

Distance (grid units)

Figure 5. Neural assemblies simultaneously coded memory strategy and journeys, and journey coding predominated in the goal arm. $\boldsymbol{A}$, Firing rate functions. Each maze arm was linearized and divided into bins. Firing rate functions were calculated as the collection of bin-wise firing rate maps for each field belonging to an assembly, defined as the set of place fields recorded simultaneously in one area of the maze (left). Difference firing functions were computed by subtracting firing rates in corresponding bins (right). $\boldsymbol{B}$, Assembly activity across conditions. An example of firing rate functions (top left) and their absolute value differences (right and bottom) are shown as 3D plots. The data represent 26 units recorded simultaneously during journeys through the north start arm. Spatial bins are on the $x$-axis, individual fields aligned as rows on the $y$-axis, and firing rates (in $\mathrm{Hz}$ ) on the $z$-axis, shown by color (blue, 0 ; dark red, $30 \mathrm{~Hz}$ ). C, Changes in firing rates across tasks or across journeys were assessed by summing the absolute values of the differences in each bin. An example of a difference firing function is shown at the bottom, with the sum of firing rates superimposed as a white line. Averaged across assemblies, these sums reflected the on-line activity changes. D, Assembly activity changes: group statistics. Mean firing rate differences in the neural population recorded simultaneously were calculated across tasks (red and green lines) and across journeys (black lines) along the length of the maze. In the center point, firing rate comparisons in behaviorally homogenous conditions were possible only across tasks (blue line). Shaded areas show $95 \%$ confidence intervals. In the start arm, memory strategy and spatial destination modulated firing rates at comparable levels. In the goal arm, origin of the journey modulated firing rate preferentially and the effect was strongest at the entry in the goal arms. Differences were larger overall in the goal arm.

$p<0.0001$; journeys: $F_{(1,2176)}=90.29, p<0.0001$, Tukey post hoc corrected).

To distinguish the influence of task and memory demands from uncontrolled factors (e.g., a drift in firing rates over time), we compared the actual rate difference functions to shuffled datasets. For across-task shuffling, the task identity of each trial for a single journey was assigned randomly to either cue or spatial. For across-journey shuffling, the journey identity of each trial sharing a common arm within a task (i.e., NE, NW) was assigned randomly. The shuffled trials were compiled into surrogate session means by averaging the corresponding datasets. The actual differences across tasks and journeys were significantly higher than the shuffled data, showing that the results were due to experimental treatments and not other uncontrolled factors (task: start arm: $F_{(1,63)}=58.6, p<0.0001$; center point: $F_{(1,109)}=37.16, p<0.0001$; goal arm: $F_{(1,65)}=83.05, p<0.0001$; journeys: start arm: $F_{(1,63)}=42.51, p<0.0001$; goal arm: $\left.F_{(1,65)}=92.65, p<0.0001\right)$. Thus, at the start of journeys and before the choice point, memory strategy and journey destination modulated the activity of neural assemblies at similar levels. However, as the rat moved past the choice point, the origin of the journey predominated. This result confirmed that the changes in firing rate of journey-dependent fields revealed by ANOVA correspond to dynamic changes in hippocampal population activity as the animals performed the tasks.

Population vector correlations verified these observations. Pearson's $r$ was computed for each corresponding pair of firing rate functions to compare the bin-wise mean firing rate along the start or goal arm (as described above to calculate rate differences). Across journeys, the population vector correlations were typically higher in the start than in the goal $\left(t_{(126)}=\right.$ 5.1, $p<0.0001)$ while across tasks, the population vector correlations were simi$\operatorname{lar}\left(t_{(124)}=0.58, p<0.56\right)$. Moreover, in the start arm, the distributions of population vector correlations comparing task and journey were similar $\left(t_{(123)}=0.06\right.$, $p<0.95)$, while in the goal arm, the corresponding distributions differed $\left(t_{(127)}=\right.$ 3.91, $p<0.0001)$, showing again that population coding distinguished the history of different journeys more than memory strategies.

\section{Discussion}

Our results demonstrate a direct link between memory demands and journeydependent activity in animals performing behaviorally identical but cognitively distinct tasks in a plus maze. Before exiting the choice point, when rats had to select a memory strategy, cells with journeydependent fields in one task were as likely as not to have journeyindependent fields in the other task. After the choice point, however, when memory strategy had been selected, most place fields had retrospective activity that was consistent across tasks. In the start arms, the firing rates in journey-dependent fields 
varied with memory strategy and journey destination to a similar degree. In the goal arm, however, firing rates varied more strongly with journey origin than memory strategy. Together, the results provide definitive evidence that differential firing of hippocampal neurons reflects memory demand and suggest that temporally extended episodes are encoded by both population and firing rate codes.

\section{Episodes may be encoded by both population and firing rate codes}

An important and unexpected finding of this study is that population and firing rates coding appear to provide two different memory signals in the same spatial environment. Before the choice point, when memory strategy was crucial for guiding behavior, different groups of place fields showed discriminative activity, suggesting that strategies were signaled by population coding. After the choice point, when the memory strategy was not important for guiding behavior, firing rates and not population codes tended to signal the journey origin. The data do not establish whether the relative influence of journey versus memory task on firing rates is due to the increased importance of representing the end of a behavioral episode, a decreased importance of representing memory strategy after the behavioral choice has been made, or both. However, the results are consistent with recent models of dentate gyrus function that propose a dual rate and population code for representing behavioral episodes (RennóCosta et al., 2010) and support the previous finding that when animals explore distinct enclosures in the same location, firing rates change but place field locations do not (rate remapping) (Leutgeb et al., 2005). Thus, our data support the idea that nonspatial changes in the environment are coded by changes in firing rates, and additionally demonstrate that rate remapping occurs not only when sensory, but also when cognitive experiences unrelated to spatial factors are discriminated at the same position in the environment. The data show that place fields do not simply map the same space whether or not the hippocampus is needed to guide task performance. Rather, hippocampal neurons generate distinct representations depending on task demands. Beyond location, trajectory, and spatial goals, both memory strategy and content alter hippocampal codes.

\section{When is journey-dependent coding present?}

The identity of all factors that influence journey-dependent coding remains unclear, but converging data suggest a link between cognitive demand and discriminative hippocampal activity. Journey-dependent coding was prominent when rats performed a hippocampus-dependent matching to place task in a plus maze, but the same CA1 neurons had journey-independent place fields when the rats foraged randomly in the same apparatus (Smith and Mizumori, 2006). When different internal states guided memory to select among visual cues, hippocampal neurons distinguished those states (Kennedy and Shapiro, 2009). Differential firing was rare during right- and left-turn trials on the central stem of a T-maze when rats performed a hippocampusdependent delay-nonmatch-to-place task, but the same population of CA1 neurons strongly discriminated sample and choice trials (Griffin et al., 2007), the aspect of the task most relevant to performance. No journey-dependent coding was found in a cue approach, win-stay task (Berke et al., 2009), or when rats were trained to follow spatial trajectories signaled initially by a flashing light, which encourage dorsal striatum-dependent strategies (McDonald and White, 1993). Thus, discriminative hippocampal activity cannot be explained by stereotyped movement or merely walking to different locations in an environment, as previously hypothesized (cf. O’Keefe, 2007). Together, these data show that differential firing in the same location by hippocampal neurons encodes the features of situations that define and potentially guide memory-based discriminations (Eichenbaum et al., 1999).

The precise timing of memory retrieval may determine whether and when journey-dependent coding is observed in the hippocampus. Although cognitive demand altered journeydependent coding, switching between hippocampus-dependent and hippocampus-independent tasks did not alter the proportions of prospective and retrospective fields. This result contrasts with those obtained in spatial alternation tasks using a modified T-maze. Differential CA1 activity on the central stem predicted choices when the animals performed a hippocampus-independent continuous alternation task (Wood et al., 2000), but when delays were introduced between trials so that the task became hippocampus-dependent, most differential firing occurred during the delay interval when the rat was confined in a box (Ainge et al., 2007b) or running wheel (Pastalkova et al., 2008). "Waiting in a box" between trials cannot be the determining factor, because in our plus maze tasks animals also waited between trials on a side platform. Furthermore, when rats were trained to approach one of four goals in a concatenated $\mathrm{Y}$-maze, CA1 activity discriminated pending choices in the start arms, rather than during the $10 \mathrm{~s}$ interval spent in a waiting box at the beginning of the trials (Ainge et al., 2007a). These conflicting results may be resolved by considering precisely when memory retrieval occurs during the different tasks. If prospective coding reflects active memory retrieval, then retrieval and code activation should coincide. In different experiments, prospective coding may vary because retrieval can occur at different times. In the current task, the rat cannot predict which starting point or journey will be used in the next trial until after it is placed on the start arm. Thus, memory retrieval that can guide performance must occur between the time the rat is placed on the start arm and the time it exits the choice point, precisely when we observed strong prospective signals. In contrast, in the spatial alternation task in which rats are confined between trials at the bottom of the central stem or in a running wheel, the animals know the future destination throughout the continuing journeys, including while waiting, when differential activity is recorded. From this perspective, the results are consistent, and suggest that differential activity of hippocampal neurons is related to memory discriminations.

The similarity in proportions of prospective, retrospective, and journey-independent fields across tasks clarified other aspects of the link between journey-dependent coding and behavior. First, prospective coding is not likely to signal blocks of trials (Morris, 2007) or behavioral context (Smith and Mizumori, 2006) because it is present whether the same goal was presented in blocks or in pseudorandom sequences. Second, journeydependent coding can occur when rats perform a task that does not require the hippocampus, supporting the idea that the hippocampus can be on line even when its function is not required (Morris and Frey, 1997). This observation fits with other experiments showing that the hippocampus encodes the regularities of tasks automatically, including those showing that hippocampal neurons model the temporal sequence of associations during delayed eyeblink conditioning, another hippocampus-independent task (Berger et al., 1983). These observations suggest that the hippocampus may encode event sequences in various types of circumstances, perhaps to allow 
more rapid adaptation to similar situations that do require the structure, such as when tasks acquire integrating information over longer intervals, e.g., in trace conditioning (McEchron et al., 2003). Third, journey-dependent coding declined equivalently during error trials in both the place and the cue approach tasks, showing that hippocampal activity is tightly correlated with memory discriminations even when the structure is not required for the performance. Perhaps this finding should not be surprising, as it dovetails with an extensive literature on interactions among memory systems (Kim and Baxter, 2001). Lesions of the hippocampus can improve caudate-dependent stimulus-approach learning, suggesting that hippocampal activity may interfere with the contribution of dorsal striatal circuitry to behavior (McDonald and White, 1993; White and McDonald, 2002). The present results show that journey miscoding correlates with cue-approach errors, and suggest future, dual recording site experiments to investigate how hippocampal activity might influence learning and memory that requires other brain structures.

\section{Hippocampus encodes journeys together with circumstances within which they occur}

CA1 journey-dependent firing patterns encoded memory strategy along with other defining task features. Nonetheless, spatial location was more strongly encoded by place fields than memory strategy or journey type, particularly in the goal arm, making space the primary factor represented. Similarly, in an object recognition memory task, location coding was more prominent than object coding in a task that required object recognition memory (Manns and Eichenbaum, 2009). The results were interpreted as showing that objects were nodes in a spatial cognitive map that helped identify objects in space. Our and others' results (Kennedy and Shapiro, 2009), however, suggest that relevant aspects of experience do not have to be punctuated objects or nodes embedded in a spatial grid. Rather, the representation is more akin to a memory space (Eichenbaum et al., 1999) in which temporally extended representations are formed by sequential coding of events. We previously hypothesized that journey-dependent fields can be considered event and sequence representations, while journey-independent fields may represent nodes that link episodes with common elements (Ferbinteanu and Shapiro, 2003). The present and previous (Kennedy and Shapiro, 2009) results extend these findings. Journey-dependent fields do not simply encode trajectories, a succession of physical locations, but rather journeys between a starting point and a behavioral goal. The journey and its hippocampal representation are modulated by internal factors, including motivation and memory strategy, to guide overt behavior.

In summary, the results show a direct link between memory, hippocampal journey-dependent activity, and place fields firing rates. The data support the idea that rate remapping may reflect the simultaneous coding of spatial and nonspatial factors and suggest that the hippocampus employs both population and firing rate codes to represent episodes.

\section{References}

Ainge JA, Tamosiunaite M, Woergoetter F, Dudchenko PA (2007a) Hippocampal CA1 place cells encode intended destination on a maze with multiple choice points. J Neurosci 27:9769-9779.

Ainge JA, van der Meer MA, Langston RF, Wood ER (2007b) Exploring the role of context-dependent hippocampal activity in spatial alternation behavior. Hippocampus 17:988-1002.

Berger TW, Rinaldi PC, Weisz DJ, Thompson RF (1983) Single-unit analysis of different hippocampal cell types during classical condition- ing of rabbit nictitating membrane response. J Neurophysiol 50:1197-1219.

Berke JD, Breck JT, Eichenbaum H (2009) Striatal versus hippocampal representations during win-stay maze performance. J Neurophysiol 101: $1575-1587$.

Bower MR, Euston DR, McNaughton BL (2005) Sequential-contextdependent hippocampal activity is not necessary to learn sequences with repeated elements. J Neurosci 25:1313-1323.

Buckner RL (2010) The role of the hippocampus in prediction and imagination. Annu Rev Psychol 61:27-28.

Eichenbaum H, Dudchenko P, Wood E, Shapiro M, Tanila H (1999) The hippocampus, memory, and place cells: is it spatial memory or a memory space? Neuron 23:209-226.

Ferbinteanu J, Shapiro ML (2003) Prospective and retrospective memory coding in the hippocampus. Neuron 40:1227-1239.

Fox SE, Ranck JB Jr (1981) Electrophysiological characteristics of hippocampal complex-spike cells and theta cells. Exp Brain Res 41: $399-410$.

Frank LM, Brown EN, Wilson M (2000) Trajectory encoding in the hippocampus and entorhinal cortex. Neuron 27:169-178.

Gray CM, Maldonado PE, Wilson M, McNaughton B (1995) Tetrodes markedly improve the reliability and yield of multiple single-unit isolation from multi-unit recordings in cat striate cortex. J Neurosci Methods 63:43-54.

Griffin AL, Eichenbaum H, Hasselmo ME (2007) Spatial representations of hippocampal CA1 neurons are modulated by behavioral context in a hippocampus-dependent memory task. J Neurosci 27:2416-2423.

Howell DC (1997) Statistical methods for psychology. Belmont, CA: Duxbury.

Kennedy PJ, Shapiro ML (2009) Motivational states activate distinct hippocampal representations to guide goal-directed behaviors. Proc Natl Acad Sci U S A 106:10805-10810.

Kim JJ, Baxter MG (2001) Multiple brain-memory systems: the whole does not equal the sum of its parts. Trends Neurosci 24:324-330.

Kim SM, Frank LM (2009) Hippocampal lesions impair rapid learning of a continuous spatial alternation task. PLoS One 4:e5494.

Kwan D, Carson N, Addis DR, Rosenbaum RS (2010) Deficits in past remembering extend to future imagining in a case of developmental amnesia. Neuropsychologia 48:3179-3186.

Leutgeb S, Leutgeb JK, Barnes CA, Moser EI, McNaughton BL, Moser MB (2005) Independent codes for spatial and episodic memory in hippocampal neuronal ensembles. Science 309:619-623.

Manns JR, Eichenbaum H (2009) A cognitive map for object memory in the hippocampus. Learn Mem 16:616-624.

McDonald RJ, White NM (1993) A triple dissociation of memory systems: hippocampus, amygdala, and dorsal striatum. Behav Neurosci 107:3-22.

McEchron MD, Tseng W, Disterhoft JF (2003) Single neurons in CA1 hippocampus encode trace interval duration during trace heart rate (fear) conditioning in rabbit. J Neurosci 23:1535-1547.

Morris R (2007) Theories of hippocampal function. In: The hippocampus book (Andersen P, Morris RG, Amara DA, Bliss T, O’Keefe J, eds), pp 581-713. Oxford: Oxford UP.

Morris RG, Frey U (1997) Hippocampal synaptic plasticity: role in spatial learning or the automatic recording of attended experience? Philos Trans R Soc Lond B Biol Sci 352:1489-1503.

O'Keefe J (1979) A review of the hippocampal place cells. Prog Neurobiol 13:419-439.

O’Keefe J (2007) Hippocampal neurophysiology in the behaving animal. In: The hippocampus book (Andersen P, Morris RG, Amara DA, Bliss T, O'Keefe J, eds), pp 475-548. Oxford: Oxford UP.

O’Keefe J, Dostrovsky J (1971) The hippocampus as a spatial map: preliminary evidence from unit activity in the freely-moving rat. Brain Res 34:171-175.

Packard MG, McGaugh JL (1996) Inactivation of hippocampus or caudate nucleus with lidocaine differentially affects expression of place and response learning. Neurobiol Learn Mem 65:65-72.

Pastalkova E, Itskov V, Amarasingham A, Buzsáki G (2008) Internally generated cell assembly sequences in the rat hippocampus. Science 321:1322-1327.

Ranck JB Jr, Kubie JL, Fox SE, Wolfson S, Muller RU (1982) Single neuron 
recording in behaving mammals: bridging the gap between neuronal events and sensory-behavioral variables. In: Behavioral contributions to brain research (Robinson TE, ed). Oxford: Oxford UP.

Rennó-Costa C, Lisman JE, Verschure PF (2010) The mechanism of rate remapping in the dentate gyrus. Neuron 68:1051-1058.

Rosenbaum RS, Gilboa A, Levine B, Winocur G, Moscovitch M (2009) Amnesia as an impairment of detail generation and binding: evidence from personal, fictional, and semantic narratives in K.C. Neuropsychologia 47:2181-2187.

Smith DM, Mizumori SJ (2006) Learning-related development of contextspecific neuronal responses to places and events: the hippocampal role in context processing. J Neurosci 26:3154-3163.

Tulving E (1972) Episodic and semantic memory. In: Organization of memory (Tulving E, Donaldson W, eds), pp 382-403. New York: Academic.

Tulving E (2001) Episodic memory and common sense: how far apart? Philos Trans R Soc Lond B Biol Sci 356:1505-1515.

Tulving E (2002) Episodic memory: from mind to brain. Annu Rev Psychol 53:1-25.

Tulving E, Markowitsch HJ (1998) Episodic and declarative memory: role of the hippocampus. Hippocampus 8:198-204.

White NM, McDonald RJ (2002) Multiple parallel memory systems in the brain of the rat. Neurobiol Learn Mem 77:125-184.

Wood ER, Dudchenko PA, Robitsek RJ, Eichenbaum H (2000) Hippocampal neurons encode information about different types of memory episodes occurring in the same location. Neuron 27:623-633. 